\title{
Ethoflow: computer vision and artificial intelligence-based software for automatic behavior analysis
}

11 * Corresponding author: R.C. Bernardes;

12 E-mail addresses: rodrigo.bernardes@ufv.br

13 ORCID: https://orcid.org/0000-0001-9481-036X

\section{Abstract}

1. Manual monitoring of animal behavior is time-consuming and prone to bias. An alternative to such

17 limitations is the use of computational resources in behavioral assessments, such as a tracking system, to

18 facilitate accurate and long-term evaluations. There is a demand for robust software that addresses

19 analysis in heterogeneous environments (such as in field conditions) and evaluates multiple individuals

20 in groups while maintaining their identities.

21 2. The Ethoflow software was developed using computer vision and artificial intelligence (AI) tools to

22 automatically monitor various behavioral parameters. A state-of-the-art object detection algorithm based

23 on instance segmentation was implemented, allowing behavior monitoring in the field under

24 heterogeneous environments. Moreover, a convolutional neural network was implemented to assess

25 complex behaviors, thus expanding the possibilities of animal behavior analyses.

26 3. The heuristics used to automatically generate training data for the AI models are described, and the

27 models trained with these datasets exhibited high accuracy in detecting individuals in heterogeneous 
28 environments and assessing complex behavior. Ethoflow was employed for kinematic assessments and

29 to detect trophallaxis in social bees. The software runs on the Linux, Microsoft Windows, and IOS

30 operating systems with an intuitive graphical interface.

31 4. In the Ethoflow algorithm, the processing with AI is separate from the other modules, which facilitates

32 kinematic measurements on an ordinary computer and the assessment of complex behavior on machines

33 with graphics processing units (GPUs). Thus, Ethoflow is a useful support tool for applications in biology

34 and related fields.

36 Keywords: animal monitoring, convolutional neural networks, deep learning, machine learning, object

37 detection, tracking

\section{Introduction}

Behavioral studies are critical to understanding the fundamental aspects of animal ecology

41 (Anderson \& Perona, 2014; Dell et al., 2014). The assessment of animal behavior by visual inspection is

42 limited and subjective, and does not allow observations over long periods (Noldus, Spink, \&

43 Tegelenbosch, 2002). The use of computational tools in behavioral assessments, such as automatic

44 tracking systems, allows accurate and long-term evaluations of animals (Dell et al., 2014; Valletta,

45 Torney, Kings, Thornton, \& Madden, 2017). Calculation of important kinematic measurements,

46 including the tracked distance, is feasible when tracking animals, and the evaluation of complex

47 behaviors can provide relevant insights about animal biology. For example, the evaluation of complex

48 behaviors among social insects, such as changes in grooming and trophallaxis, is important for

49 understanding their response to stress agents such as pesticides (Gandra, Amaral, Couceiro, Della Lucia,

50 \& Guedes, 2016; Boff, Friedel, Mussury, Lenis, \& Raizer, 2018). 
Robust systems are needed for animal monitoring in heterogeneous environments, such as in the

52 field (Dell et al., 2014). The greatest challenge in heterogeneous environments involves the extraction of

53 target objects from the background (segmentation) (Zou, Shi, Guo, \& Ye, 2019). Animal tracking

54 software operates by background subtraction or thresholding (Yamanaka \& Takeuchi, 2018; Sridhar,

55 Roche, \& Gingins, 2019). As these approaches require video recordings with sufficient contrast between

56 the object and homogeneous background, they are not applicable in the field. Software using artificial

57 intelligence (AI) can be sufficiently robust for assessments in heterogeneous environments, as AI models

58 can be trained to learn the detection of target objects in different environments (He, Gkioxari, Dollár, \&

59 Girshick, 2018).

60 Convolutional neural networks (CNNs) are the most conventional AI models employed in

61 computer vision tasks. While these models have exhibited outstanding performance in computer vision

62 applications and tracking software, including idtracker.ai and DeepLabCut, which use AI models in their

63 algorithms, they still exhibit some limitations. The idtracker.ai software applies a CNN to maintain the

64 identity of individuals in a group, but its application is limited to homogeneous environments (Romero-

65 Ferrero, Bergomi, Hinz, Heras, \& de Polavieja, 2019). While DeepLabCut uses a CNN for animal pose

66 estimation and tracks parts of objects in heterogeneous environments (Nath et al., 2019), it does not

67 maintain the identity of individuals in a group.

Given the potential applications of $\mathrm{AI}$ and the demand for a robust system that fulfills the

69 requirement for studying animal behavior (Dell et al., 2014), the Ethoflow software was developed. AI

70 was incorporated into Ethoflow for object detection, enabling evaluations of the complex behaviors of

71 individuals in groups living in heterogeneous environments. To validate Ethoflow, bioassays with two

72 species of eusocial bees were performed. In addition, parameters associated with the performance of the

73 Ethoflow software were evaluated during its execution. 


\section{Software features}

The Ethoflow software runs on Linux, Microsoft Windows, and IOS operating systems. This

software is registered with the Brazilian National Institute of Intelectual Property (Instituto Nacional de

77 Propriedade Industrial, INPI, Ministério da Economia, Brazil, reg. no. BR 512020 000737-6). Ethoflow

78 is user-friendly and does not require the use of line commands because of the intuitive graphical user

79 interface (GUI) (Fig. 1A). There are three tabs (Settings, Analyses, and Deep analyses) on the Ethoflow

80 GUI. Under the 'Settings' tab, the parameters can be set. Once the parameters have been set, they can be

81 saved as a protocol (as a .txt file) and loaded for the next step. When setting the parameters, the interface

allows real-time monitoring of the effects of parameter changes; for example, detected objects (i.e.,

animals) will be marked with a red mask. A region of interest (Fig. 1A; blue box on the bottom right) can

also be defined to assess how long the individuals stayed in this region. The 'Analyses' tab corresponds

to the video processing step and renders images to train the instance segmentation modelErro! Fonte de

86 referência não encontrada.. During video processing, the user can visually monitor the processing in

87 real time. Following completion of processing, the program prints the processing speed and detection

88 rate on the GUI. Under the 'Deep analyses' tab, models for complex behavior analysis are loaded, which

89 allows the recognition of several specific behaviors on the condition that an AI model is set up. The

90 median and standard deviation of some measurements of the body of individuals (for e.g., area and

91 length) can also be calculated. These parameters can serve as a basis for generating labeled images to

92 train the specific behavior model and assist in the protocol definition.

In the Ethoflow algorithm, multi-threaded reading was implemented. This avoids the delay

94 between frame reading and other processing steps of the algorithm, whereby frames are always available,

95 thus making it possible to obtain high rates in frames per second (fps) (Supporting Information A.1). In

96 addition, the AI processing is separate from the other modules in the Ethoflow algorithm. Therefore, 
97 Ethoflow enables kinematic measurements on an ordinary computer and the assessment of more complex

98 behavior using a GPU.

99 Manual and automatic thresholds were applied in Ethoflow to detect individuals. In manual

100 thresholding, the threshold value is defined by the user. One of the automatic thresholding options is

101 based on Otsu's method (Otsu, 1979), wherein the optimal threshold minimizes the within-class variance

102 (Supporting Information A.3). The other automatic thresholding option involves instance segmentation

103 (IS), which is based on the Mask R-CNN AI model (He et al., 2018). This state-of-the-art model for

104 object detection allows Ethoflow to work in field conditions, detecting individuals in a heterogeneous

105 background (Fig 1. B). The hyperparameters and architecture of the IS model are based on the

106 implementation reported by Abdulla (2017) (Supporting Information A.3). The IS model should be

107 trained to learn to detect the animal of interest. Thus, a heuristic was used to automatically generate the

108 training data. This heuristic functions by extracting the contours of individuals in a homogeneous

109 background and subsequently pasting them in a heterogeneous background. (Supporting Information

110 A.3).

111 Ethoflow monitors animals in groups, maintaining the identity of individuals. In this step of the

112 algorithm, the nonhierarchical clustering k-means is applied to separate merged individuals.

113 Subsequently, a combinatorial optimization algorithm is applied, which provides the optimal identity

114 assignment between individuals (Supporting Information A.5). Ethoflow records the coordinates of

115 movement in time and calculates various kinematic parameters associated with the behavior of

116 individuals and groups (Supporting Information A.6). Moreover, Ethoflow measures specific behaviors

117 using a CNN model (Fig. 1C); different hyperparameter configurations were tested to define the CNN

118 model, which can be used to recognize binary behaviors (Supporting Information A.7). The data used to

119 train the CNN model was also generated with a heuristic based on the animal body size and length

120 (Supporting Information A.7). 
A
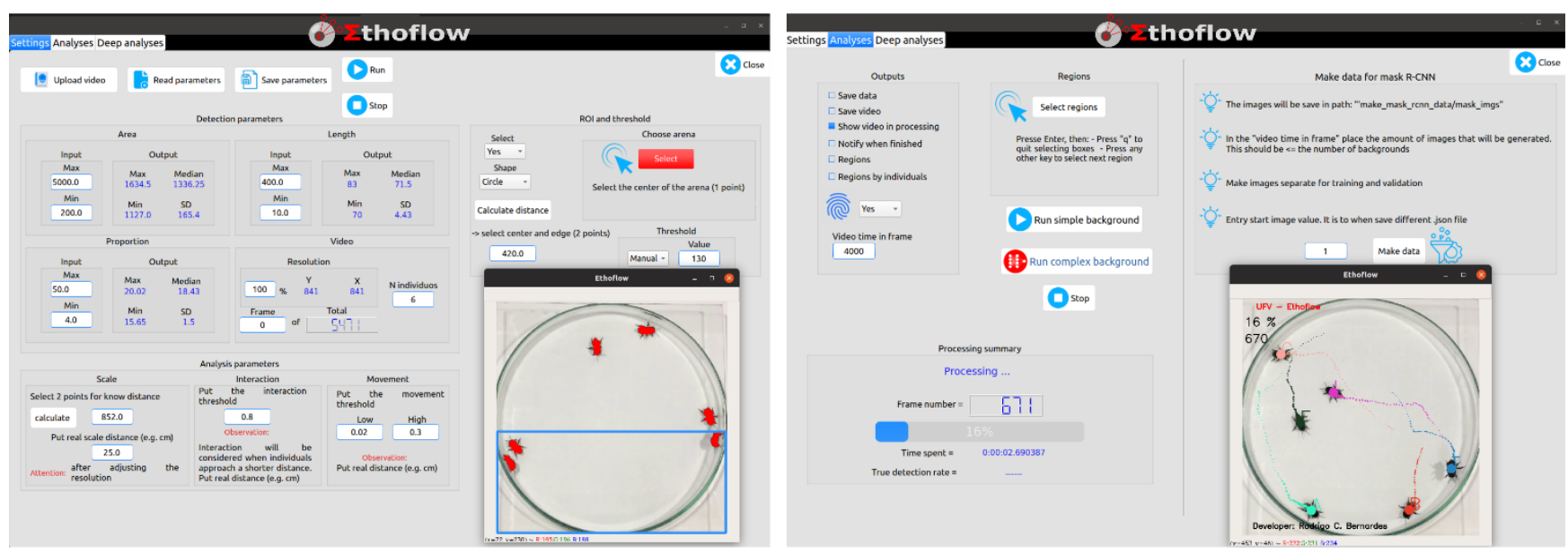

B

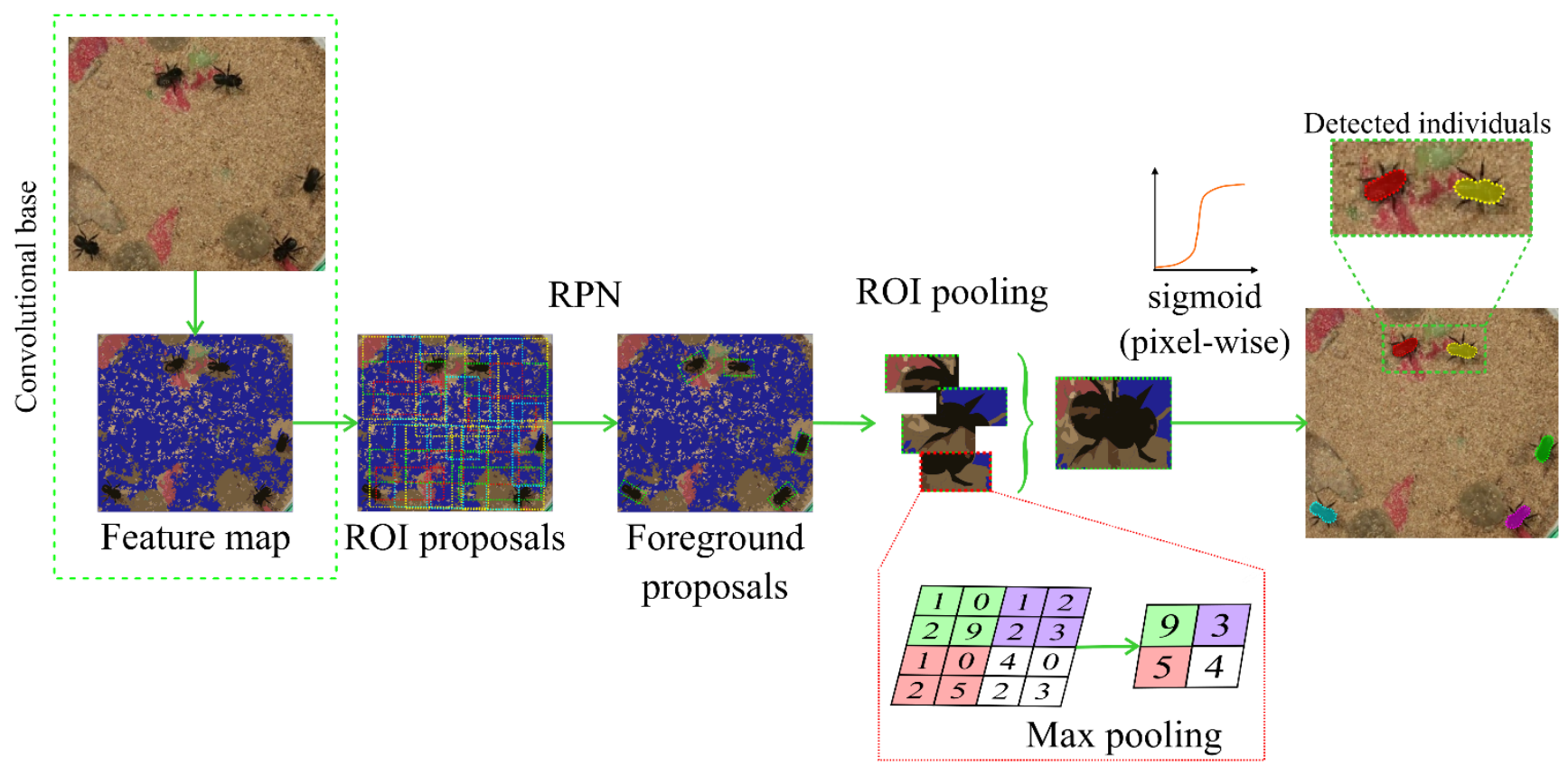

C

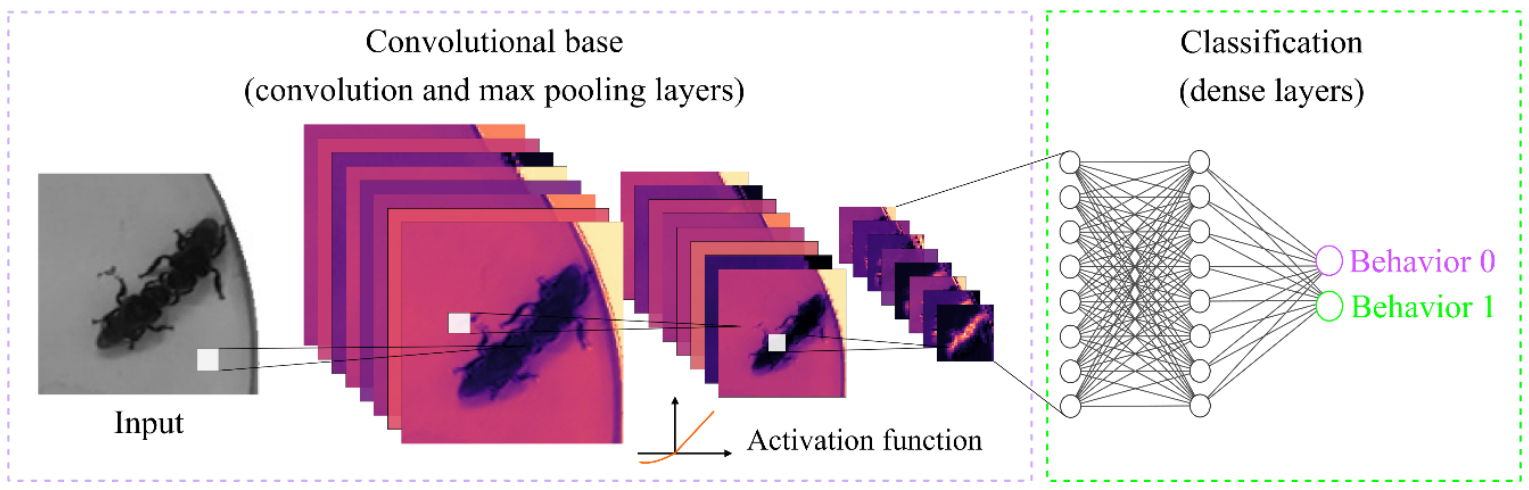


123 Figure 1. Features of the Ethoflow software. (A) Graphical user interface (GUI). In the left panel (Settings

$124 \mathrm{tab}$ ), the detected individuals are marked with a red mask after adjusting the parameters (protocol

125 definition). Blue box on the bottom right shows the selected region of interest, which is used to assess

126 how long the individuals remain inside the defined region. In the right panel (Analyses tab), monitoring

127 of the processing step is demonstrated. Each individual is randomly assigned an identity and color. In

128 this example, the analyzed objects correspond to workers of the stingless bee species Melipona

129 quadrifasciata present in a Petri dish (15 cm diameter, $2 \mathrm{~cm}$ height). (B) Diagram depicting the operations

130 involved in instance segmentation. The input passes through a convolutional base for feature extraction,

131 leading to the generation of a feature map. The region proposal network (RPN) is applied, which provides

132 several candidate boxes (ROI proposals). As several ROIs are generated, the model classifies these boxes

133 into foreground proposals (objects) and backgrounds. ROI pooling is applied to standardize the size of

134 the foreground proposals, slicing each foreground into a fixed amount of parts, and max pooling is 135 applied to standardize the size. Then, the boxes labeled as real objects (the individuals) are instantiated

136 using a pixel-wise sigmoid function. (C) Workflow of the convolutional neural networks used in

137 Ethoflow to assess complex behaviors. In the convolutional base, the input passes through the

138 convolutional and max pooling layers for feature extraction. Then, behavior classification is performed

139 in the dense layers. The activation function is applied to the output of each layer to introduce non-

140 linearity.

\section{Performance and applications}

Ethoflow was trained to detect the bee Melipona quadrifasciata in several field conditions

144 (Supporting Information B.1). The proposed model was efficient in detecting all animals in

145 heterogeneous backgrounds with high precision (average precision \pm standard error $=0.916 \pm 0.02 ;$ Fig.

146 2A). Ethoflow was also trained to learn the detection of trophallaxis, the complex social behavior of food 
bioRxiv preprint doi: https://doi.org/10.1101/2020.07.23.218255; this version posted July 24, 2020. The copyright holder for this preprint (which was not certified by peer review) is the author/funder, who has granted bioRxiv a license to display the preprint in perpetuity. It is made available under aCC-BY-NC-ND 4.0 International license.

147 exchange among nestmates, in M. quadrifasciata (Supporting Information B.1). This model exhibited

148 high accuracy in the validation process (global accuracy $=92.13 \%$, Kappa index $=0.84, Z=24.74$, Fig.

149 2B). 
bioRxiv preprint doi: https://doi.org/10.1101/2020.07.23.218255; this version posted July 24, 2020. The copyright holder for this preprint (which was not certified by peer review) is the author/funder, who has granted bioRxiv a license to display the preprint in perpetuity. It is made available under aCC-BY-NC-ND 4.0 International license.

A
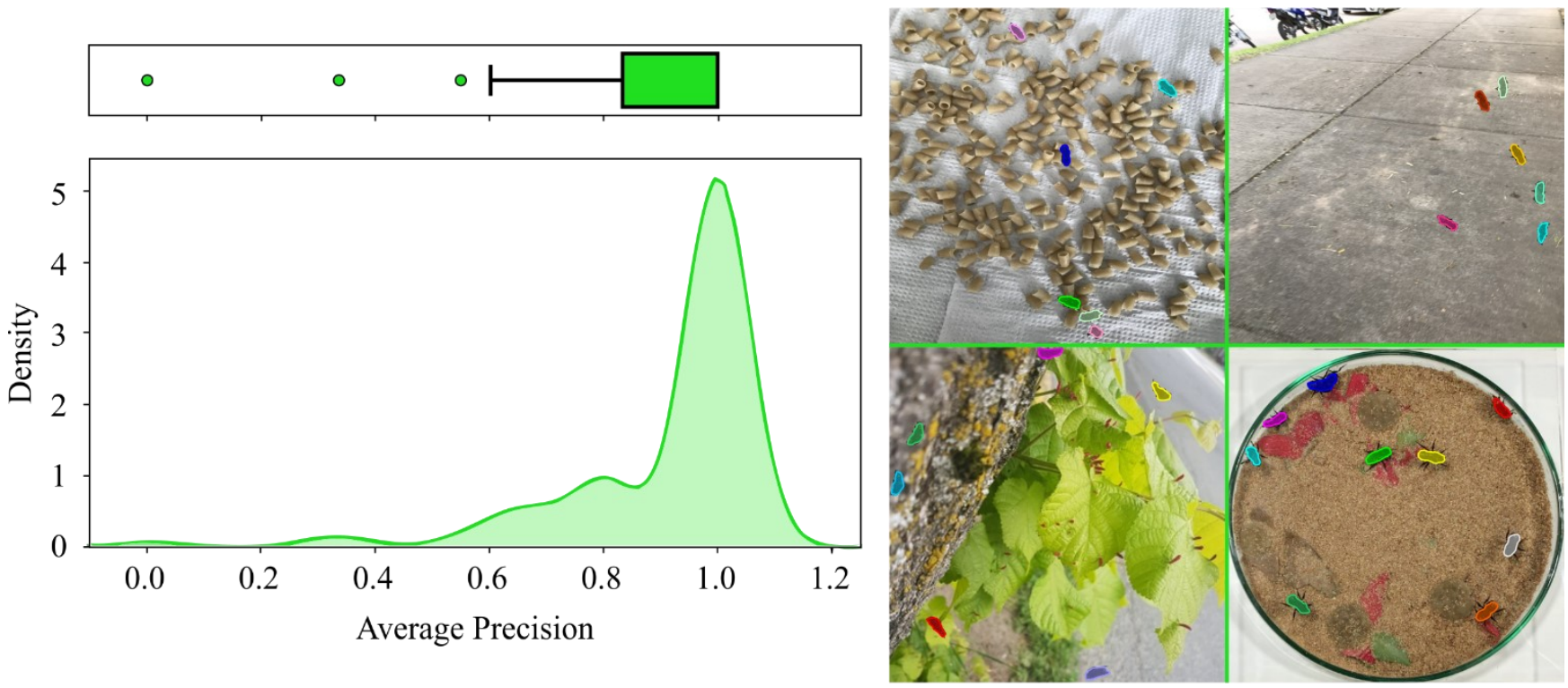

B
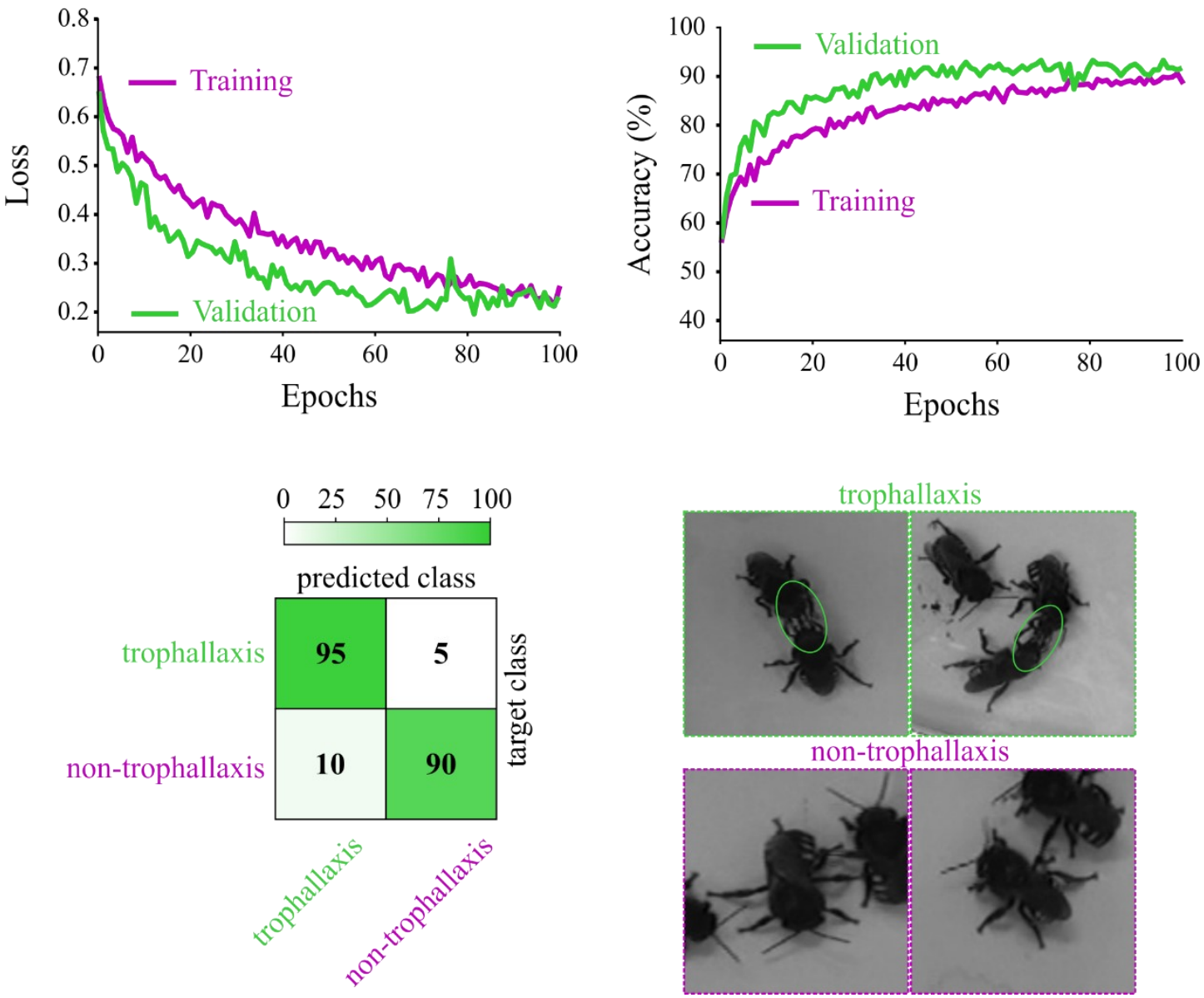
153 Figure 2. Performance of the AI models used in Ethoflow. (A) Object detection in heterogeneous

154 backgrounds based on instance segmentation (IS). The high average precision (left panel; $n=100$ )

155 implies that the model precisely detects real objects in the scenes with no false positives, as demonstrated

156 by (right panel) the detected objects (Melipona quadrifasciata bees) with masks in random colors. (B)

157 The training process of the CNN model (top panel) and cross-validation (confusion matrix; bottom left 158 panel) $(n=127)$ for the monitoring of trophallaxis (green circles) in bees.

To validate Ethoflow, a behavioral assay was performed with the stingless bee species $M$.

161 quadrifasciata and Partamona helleri (Supporting Information B.2) and different kinematic variables

162 were measured (definitions of the variables are detailed in Supporting Information A.6). In both species,

163 the centrality increased with the polarization of the group $\left(\mathrm{F}_{1,35}=25.1, p<0.0001\right)$ and decreased with

164 milling $\left(\mathrm{F}_{1,35}=46.2, p<0.0001\right)$ (Fig. 3A). Meandering was influenced by the statistical interaction

165 between the variables resting and bee species $\left(\mathrm{F}_{1,33}=4.71, p=0.037\right.$; Fig. 3B). Moreover, a difference

166 between species was observed in the tracked distance $\left(\mathrm{F}_{1,35}=13.6, p=0.0008\right.$; Fig. 3C).

A toxicological bioassay was performed with M. quadrifasciata to demonstrate the recognition

168 of trophallaxis under pesticide stress conditions. The pesticide imidacloprid, which is usually associated

169 with the decline of bees, was used (Lima, Martins, Oliveira, \& Guedes, 2016). The exposure protocol

170 was based on that reported by Botina et al. (2020) (Supporting Information B.2). Bees exposed to the

171 pesticide exhibited significantly reduced trophallaxis $\left(\chi^{2}=94.9\right.$, $\mathrm{df}=58, p<0.0001$; Fig. 3D). 
bioRxiv preprint doi: https://doi.org/10.1101/2020.07.23.218255; this version posted July 24, 2020. The copyright holder for this preprint (which was not certified by peer review) is the author/funder, who has granted bioRxiv a license to display the preprint in perpetuity. It is made available under aCC-BY-NC-ND 4.0 International license.
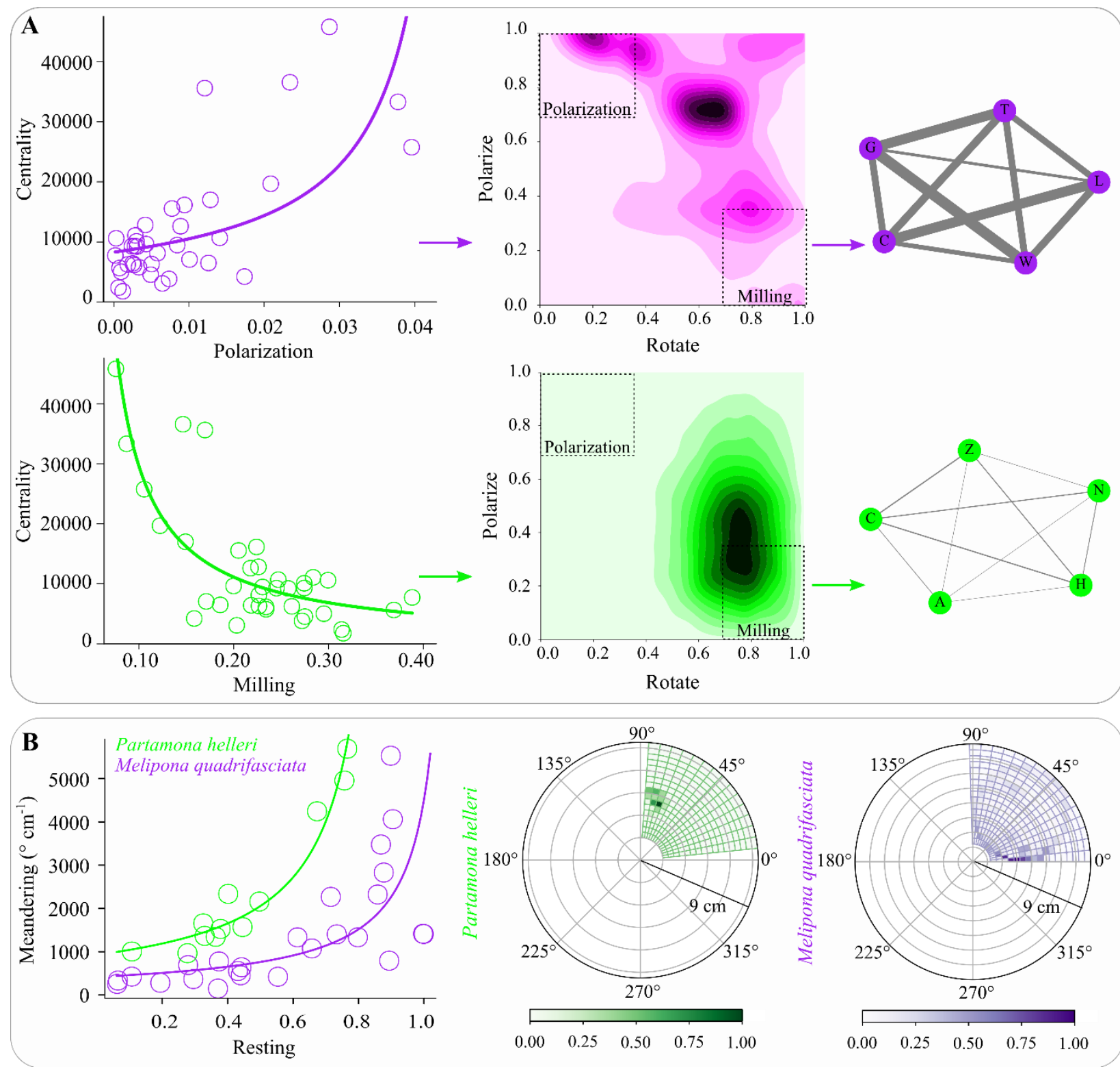

C

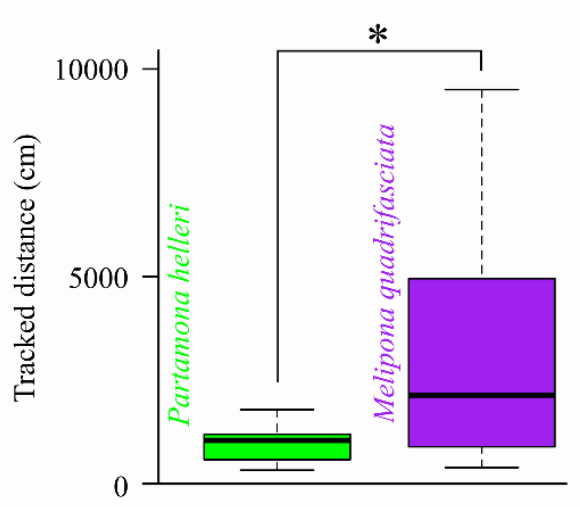

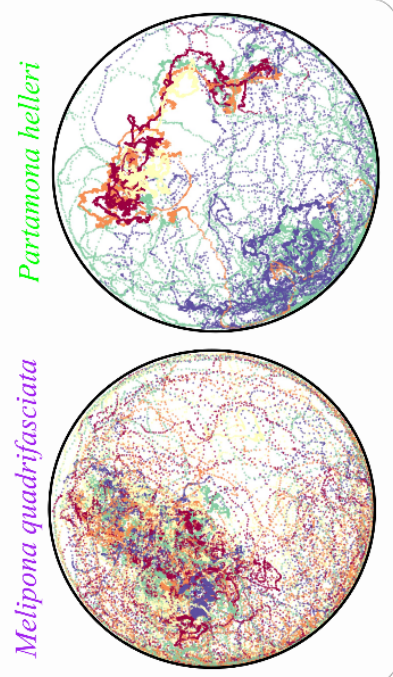

D
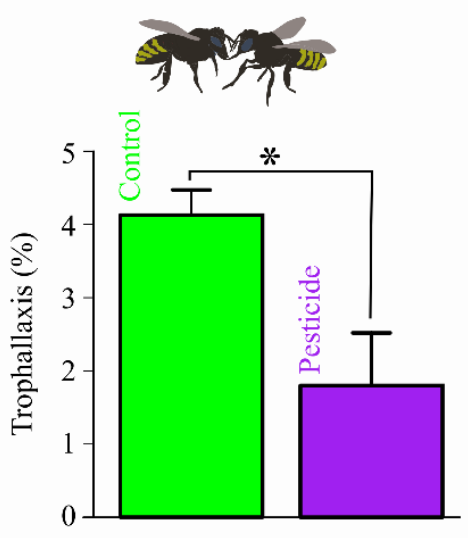
174 Figure 3. Behavioral assessments conducted using the Ethoflow software. (A) Association between

175 centrality and group dynamics polarization (top panel) and milling (bottom panel) $(n=37)$. The 2D

176 density plots and network diagrams showed that in more polarized bee groups, a higher interaction exists

177 among individuals, while this interaction is reduced in the milling groups. In the networks, the circles

178 represent individuals and connections correspond to the edges, where their widths are proportional to the

179 frequency of interactions. (B) Meandering behavior is associated with resting proportions (left panel) (n

$180=37$ ) and histograms of polar coordinates (rays and azimuth angles) for the two bee species (right panel).

181 (C) The tracked distance of the assessed bee species $(n=37)$. In group representative tracks, the track

182 color reflects the individual identity (right panel). (D) Trophallaxis alteration in M. quadrifasciata after

183 pesticide exposure $(n=60) .{ }^{*} p<0.05$ in the generalized linear model. When an explanatory variable

184 had no significant effect, the model was simplified, and the results were plotted as a function of the 185 significant variable. in the range of fps $\left(\mathrm{F}_{1,130}=12.81, p=0.0005\right.$, Fig. 4A). The heterogeneous environment quantification was not influenced by the resolution or number of individuals $\left(\mathrm{F}_{1,28}=0.81, p=0.37\right.$, Fig. $\left.4 \mathrm{~B}\right)$. The fps decreased with an increase in the centrality of individuals ( $\mathrm{F}_{1,38}=81.24, p<0.0001$, Fig. 4C). There was no significant effect on the number of individuals $\left(\mathrm{F}_{1,37}=0.009, p=0.93\right)$, and no interaction was

194 observed between the centrality and individuals $\left(\mathrm{F}_{1,36}=1.62, p=0.21\right)$. In addition, the software 195 exhibited high detection rates (Fig. 4D). A significant interaction was observed between the number of 196 individuals and type of background $\left(\mathrm{F}_{1,94}=137.85, p<0.0001\right)$, where an increase in the number of 197 individuals had a greater influence on the heterogeneous environments. A few videos processed with 198 Ethoflow are available in B.4 of the Supporting Information. 
A

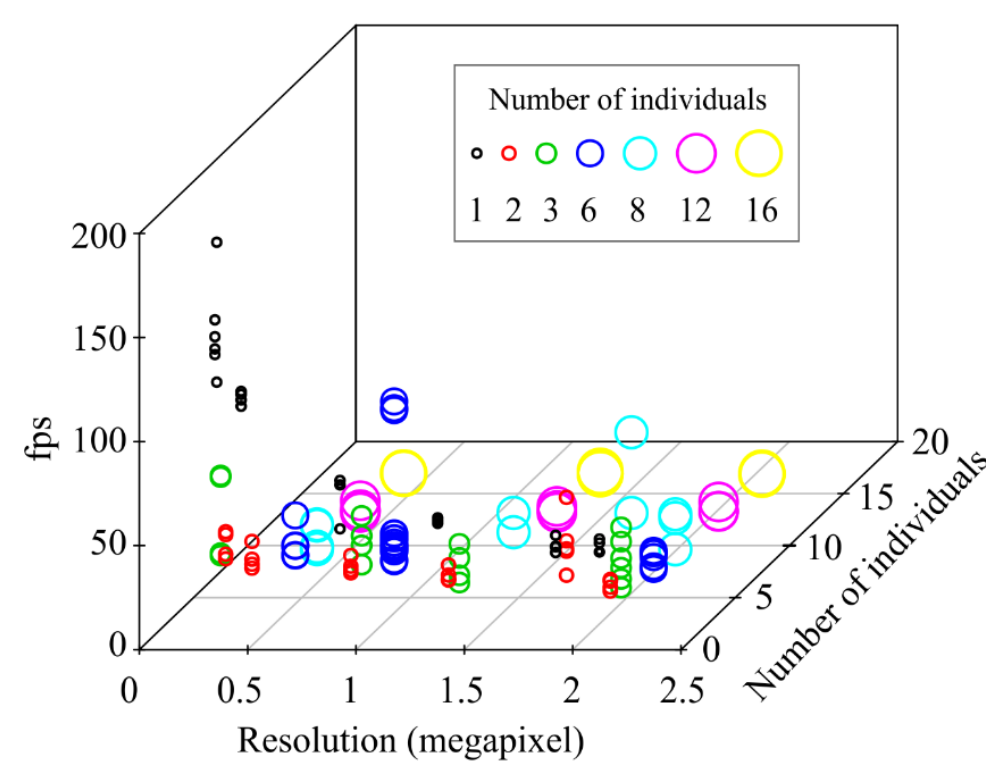

C

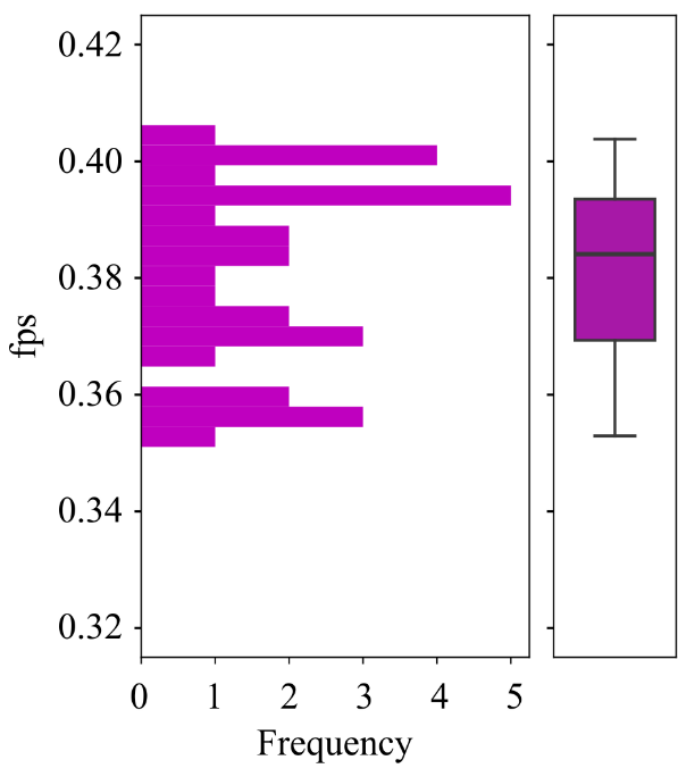

C

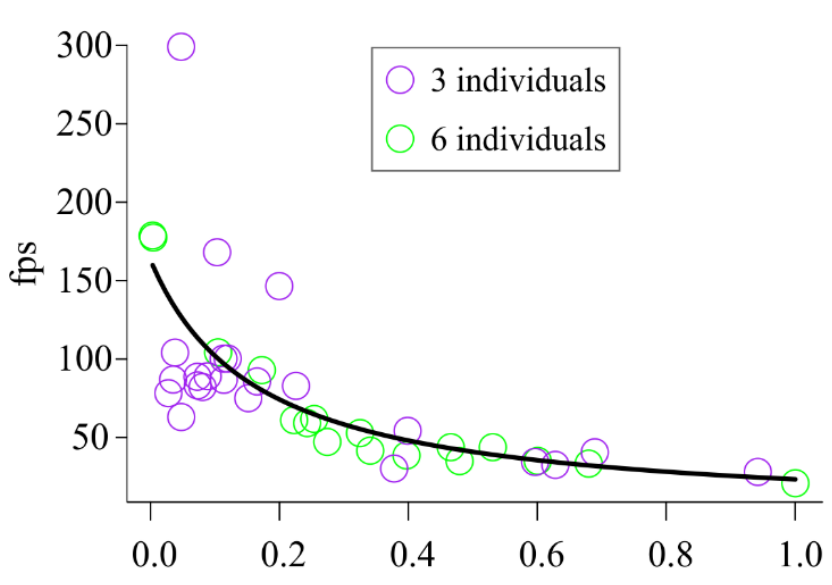

D

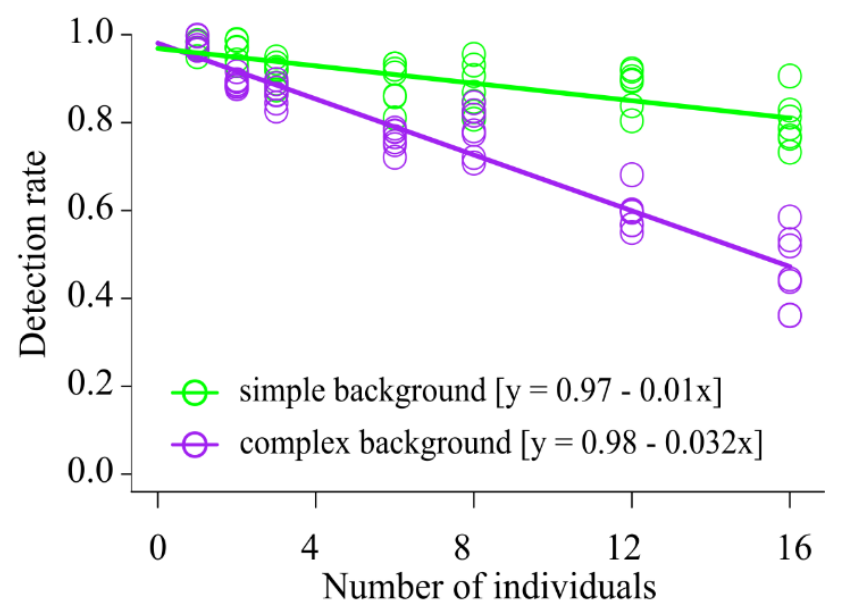

Centrality

201 Figure 4. Quantification of the performance of Ethoflow. (A) Fps in response to the video resolution (in pixels) and number of individuals in homogeneous backgrounds; the dots $(n=134)$ represent the raw data. (B) Histogram of the fps in heterogeneous environments $(n=30)$. The box plot indicates the median and range of dispersion (lower and upper quartiles and outliers). (C) Fps in response to centrality. The proportion of group interaction per frame was used to quantify the centrality $(n=40)$. (D) Accuracy of

206 the software as a function of the interaction between the number of individuals and type of environment 207 (homogeneous and heterogeneous); the symbols represent the raw data (circles; $n=98$ ). When an 
208 explanatory variable had no significant effect, the model was simplified, and the results were plotted as

209 a function of the significant variable.

2104 Conclusion

211 The Ethoflow software was developed using modern computer vision techniques and AI. This

212 software exhibited consistent speed rates and processing accuracy. The developed software is suitable

213 for behavioral assessments in heterogeneous environments, to track groups with individuals maintaining

214 their identities, and can be trained to learn specific behaviors. Ethoflow was applied to biological

215 assessments, and some possibilities of data analysis and representation were demonstrated with

216 Ethoflow's output. Accurate AI models have been implemented to expand the possibilities of animal

217 behavior analyses to other fields, including the behavioral monitoring of domestic animals in precision

218 livestock farming. Therefore, Ethoflow is a useful support tool for technical and scientific applications

219 in biology and related fields.

\section{Acknowledgements}

222 We thank the National Council of Scientific and Technological Development (CNPq 223 142206/2017-2 and 301725/2019-5) and CAPES-PROEX for financial support (Financial Code 001).

\section{Authors' contributions}

226 RCB developed the software, performed the experiments, analyzed the data; RCB and GFM 227 wrote the manuscript. All authors designed the work and contributed to the drafts and gave final approval 228 for publication.

\section{Data archiving statement}

231 The data have been archived at https://github.com/bernardesrodrigoc/Ethoflow; DOI: 232 https://doi.org/10.5281/zenodo.3956831

\section{References}


235

236

237

238

239

Abdulla, W. (2017). Mask R-CNN for object detection and instance segmentation on Keras and TensorFlow. GitHub Repository. Github.

Anderson, D. J., \& Perona, P. (2014). Toward a Science of Computational Ethology. Neuron, 84(1), 1831. doi:10.1016/J.NEURON.2014.09.005

Boff, S., Friedel, A., Mussury, R. M., Lenis, P. R., \& Raizer, J. (2018). Changes in social behavior are induced by pesticide ingestion in a Neotropical stingless bee. Ecotoxicology and Environmental Safety, 164, 548-553. doi:https://doi.org/10.1016/j.ecoenv.2018.08.061

Botina, L. L., Bernardes, R. C., Barbosa, W. F., Lima, M. A. P., Guedes, R. N. C., \& Martins, G. F. (2020). Toxicological assessments of agrochemical effects on stingless bees (Apidae, Meliponini). MethodsX, 100906. doi:https://doi.org/10.1016/j.mex.2020.100906

Dell, A. I., Bender, J. A., Branson, K., Couzin, I. D., de Polavieja, G. G., Noldus, L. P. J. J., ... Brose, U. (2014). Automated image-based tracking and its application in ecology. Trends in Ecology \& Evolution, 29(7), 417-428. doi:10.1016/J.TREE.2014.05.004

Gandra, L. C., Amaral, K. D., Couceiro, J. C., Della Lucia, T. M., \& Guedes, R. N. (2016). Mechanism of leaf-cutting ant colony suppression by fipronil used in attractive toxic baits. Pest Management Science, 72(8), 1475-1481. doi:10.1002/ps.4239

He, K., Gkioxari, G., Dollár, P., \& Girshick, R. (2018). Mask R-CNN. IEEE Transactions on Pattern Analysis and Machine Intelligence, 42(2), 386-397. doi:10.1109/TPAMI.2018.2844175

Lima, M. A. P., Martins, G. F., Oliveira, E. E., \& Guedes, R. N. C. (2016). Agrochemical-induced stress in stingless bees: peculiarities, underlying basis, and challenges. Journal of Comparative Physiology A: Neuroethology, Sensory, Neural, and Behavioral Physiology, 202(9-10), 733-747. doi:10.1007/s00359-016-1110-3

Nath, T., Mathis, A., Chen, A. C., Patel, A., Bethge, M., \& Mathis, M. W. (2019). Using DeepLabCut for 3D markerless pose estimation across species and behaviors. Nature Protocols, 14(7), 2152-2176. doi:10.1038/s41596-019-0176-0

Noldus, L. P. J. ., Spink, A. J., \& Tegelenbosch, R. A. . (2002). Computerised video tracking, movement analysis and behaviour recognition in insects. Computers and Electronics in Agriculture, 35(2-3), 201-227. doi:10.1016/S0168-1699(02)00019-4

Otsu, N. (1979). A Threshold Selection Method from Gray-Level Histograms. IEEE Transactions on Systems, Man, and Cybernetics, 9(1), 62-66. doi:10.1109/TSMC.1979.4310076

Romero-Ferrero, F., Bergomi, M. G., Hinz, R. C., Heras, F. J. H., \& de Polavieja, G. G. (2019). idtracker.ai: tracking all individuals in small or large collectives of unmarked animals. Nature Methods, 16(2), 179-182. doi:10.1038/s41592-018-0295-5 
268 Sridhar, V. H., Roche, D. G., \& Gingins, S. (2019). Tracktor: Image-based automated tracking of animal 269 movement and behaviour. Methods in Ecology and Evolution, 10(6), 815-820. doi:10.1111/2041$270 \quad 210 X .13166$

271 Valletta, J. J., Torney, C., Kings, M., Thornton, A., \& Madden, J. (2017). Applications of machine 272 learning in animal behaviour studies. Animal Behaviour, 124, $203-220$. 273 doi:https://doi.org/10.1016/j.anbehav.2016.12.005

274 Yamanaka, O., \& Takeuchi, R. (2018). UMATracker: an intuitive image-based tracking platform. The 275 Journal of Experimental Biology, 221(16).

276 Zou, Z., Shi, Z., Guo, Y., \& Ye, J. (2019). Object Detection in 20 Years: A Survey. Retrieved from 277 http://arxiv.org/abs/1905.05055 